\title{
ON A TYPE OF ALGEBRAIC DIFFERENTIAL MANIFOLD
}

\author{
BY \\ J. F. RITT
}

The manifolds $\left({ }^{1}\right)$ to be investigated, which are manifolds of systems of differential polynomials in a single unknown, possess a degree of analogy to bounded sets of numbers. They are manifolds which may be said "not to contain infinity as a solution"; more definitely, zero is not a limit of reciprocals of solutions.

For manifolds of this type, which will be called limited, operations of addition, multiplication and differentiation will be studied. Given two manifolds $\left({ }^{2}\right) \mathfrak{M}_{1}$ and $\mathfrak{M}_{2}$, their arithmetic sum is secured by completing into a manifold the totality of functions each of which is, in some area, the sum of a solution in $\mathfrak{M}_{1}$ and a solution in $\mathfrak{M}_{2}$. Multiplication is defined similarly.

It turns out that if $\mathfrak{M}_{1}$ and $\mathfrak{M}_{2}$ are general solutions of equations of the first order, and are limited, their sum and product are limited. On the other hand, as is shown by examples based on the theory of the elliptic functions, when $\mathfrak{M}_{1}$ and $\mathfrak{M}_{2}$ involve more than one arbitrary constant their limited character may not be communicated to their sum and product; what is equivalent to this, as far as multiplication is concerned, is the rather unexpected result that the product of two manifolds may contain zero even if neither manifold does.

The derivative of a limited manifold proves to be limited in all cases.

\section{LIMITED MANIFOLDS}

1. Let $\Sigma$ be a system of forms in the single unknown $y$. Let us suppose that $\Sigma$ has solutions and that it has at least one solution which is not identically zero. The transformation $z=1 / y$ carries every nonzero solution of $\Sigma$ into a definite function $z$. There exist forms in $z$ which vanish for every function $z$ thus obtained. Let $\Sigma^{\prime}$ be the totality of such forms in $z$. It is not difficult to see that the manifold of $\Sigma^{\prime}$ is the set of the reciprocals of the nonzero solutions of $\Sigma$, enlarged perhaps by the adjunction of $z=0$.

If $\Sigma^{\prime}$ does not admit $z=0$ as a solution, we shall call the manifold of the original system $\Sigma$ limited $\left({ }^{3}\right)$.

2. If $\Sigma^{\prime}$ has $z=0$ as a solution, $z=0$ cannot be an essential manifold for $\Sigma^{\prime}$. If it were, $\Sigma^{\prime}$, which is closed, would contain a form $z A$ where $A$ does not

Presented to the Society, September 12, 1940; received by the editors March 20, 1940.

(1) For indications in regard to the general theory to which this paper attaches, one may consult the author's paper in the second volume of the Semicentennial Publications of the American Mathematical Society.

(2) Not necessarily limited.

(3) If $\Sigma$ admits only $y=0$ as a solution, its manifold will also be called limited. 
vanish for $z=0$. Now $A$ would vanish for the reciprocal of every nonzero solution of the system $\Sigma$. It would thus be in $\Sigma^{\prime}$ and would rule out the solution $z=0$.

Thus, if the manifold of $\Sigma$ is not limited, there is a dense set of values of $x$ such that, given any point $a$ of the set, any positive integer $m$ and any $\epsilon>0$, we can find a solution of $\Sigma$ whose reciprocal is analytic at $a$ and has a Taylor expansion at $a$ in which the first $m+1$ coefficients have moduli less than $\epsilon$. When the manifold of $\Sigma$ is limited, no point exists which has the property, just stated, of the points $a$.

3 . Let $\Sigma$ be a closed system of forms in $y$ which admits solutions. We shall prove that for the manifold of $\Sigma$ to be limited, it is necessary and sufficient that $\Sigma$ contain a form $A$ which, considered as a polynomial in $y$ and its derivatives, possesses a term in $y$ alone, that is, a term free of the $y_{i}$ with $i>0$, which is of higher degree than every other term in $A$.

Let the manifold be limited. We may suppose that there are solutions other than $y=0$. Then $\Sigma^{\prime}$, as above, contains a form $1+K$ with $K$ a nonzero form which vanishes for $z=0$. Making the substitution $z=1 / y$ in $K$, and clearing fractions, we obtain a form in $\Sigma$ answering to the description of $A$.

Conversely, let $\Sigma$ contain a form $A$ as described. If we put $y=1 / z$ in $A$ and clear fractions, we secure a form $B$ in $\Sigma^{\prime}$, one of whose terms, free of proper derivatives of $z$, is of lower degree than every other term. Thus, if $z=0$ were in the manifold of $\Sigma^{\prime}$, it would be an essential manifold. This, by $\S 2$, is impossible.

\section{CONSIDERATIONS OF GENERAL THEORY}

4. We present here a theorem of a general character which will be employed in $\$ 10$.

Let $\Sigma$ be a nontrivial closed irreducible system in the unknowns $u_{1}, \cdots, u_{q}$; $y_{1}, \cdots, y_{p}$ with the $u_{i}$ (which may be nonexistent) arbitrary and with $p>1$. Let $m$ be any positive integer not greater than $p$. Those forms in $\Sigma$ which involve only the $u_{i}$ and $y_{1}, \cdots, y_{m}$ constitute a closed irreducible system $\Sigma_{m}$ in the unknowns just mentioned. For $m=p, \Sigma_{m}$ is $\Sigma$.

Let $m<p$. Given a solution

$$
\bar{u}_{i} ; \bar{y}_{1}, \cdots, \bar{y}_{m}
$$

of $\Sigma_{m}$, analytic in an area $\mathfrak{A}_{1}$, there may exist an area $\mathfrak{A}_{2}$ contained in $\mathfrak{A}_{1}$ and a set of functions

$$
\bar{y}_{m+1}, \cdots, \bar{y}_{p},
$$

analytic in $\mathfrak{A}_{2}$, such that (1) and (2) constitute a solution of $\Sigma$ in $\mathfrak{A}_{2}$. In that case, we shall say that the solution (1) of $\Sigma_{m}$ can be completed into a solution of $\Sigma$.

We are going to prove that there exists a form $G$ in $u_{1}, \cdots, u_{q} ; y_{1}, \cdots, y_{m}$ 
which does not belong to $\Sigma_{m}$ and which has the property that every solution of $\Sigma_{m}$ which does not annul $G$ can be completed into a solution of $\Sigma$.

5. Let

$$
A_{1}, \cdots, A_{p}
$$

be a basic set of $\Sigma, A_{i}$ introducing $y_{i}$. Let the order of $A_{i}$ in $y_{i}$ be $r_{i}$. Let $S_{i}$ and $I_{i}$ be respectively the separant and initial of $A_{i}$.

We consider the system of forms

$$
A_{1}, \cdots, A_{m+1},
$$

which is a basic set of $\Sigma_{m+1}$. Let a form $L$ be given which is not in $\Sigma_{m+1}$ and which is such that every $y_{i j}$ appearing in $L$ has $i \leqq m+1$ and $j \leqq r_{i}$. We place no restrictions on the $u_{i j}$ in $L$. We shall establish a relation

$$
R=M+N L S_{m+1}
$$

of the following description. The $y_{i j}$ in $R, M$ and $N$ have $i \leqq m+1$ and $j \leqq r_{i}$. $M$ is contained in $\Sigma_{m+1}$. $R$, distinct from zero, is free of the $y_{i r_{i}}$. Thus $R$ is not in $\Sigma_{m+1}$.

6. Let (4) be considered as a set of simple forms. Then (4) will be a basic set of a prime system( $\left.{ }^{4}\right) \Pi$. Now $L S_{m+1}$ (simple form) is not in $\Pi$. Then every indecomposable system held by $\Pi+L S_{m+1}$ has fewer unconditioned unknowns than $\Pi$. There exists thus a relation (5) with all forms simple forms, $R$ being distinct from zero and free of the $y_{i r_{i}}$, and $M$ belonging to $\Pi$. It remains only to consider the forms in (5) as differential polynomials.

7. Let

$$
J=S_{1} \cdots S_{m} I_{1} \cdots I_{m+1} R .
$$

Let $J$ be considered as a polynomial in the $y_{m+1, j}$, with coefficients which are forms in the $u_{i}$ and $y_{1}, \cdots, y_{m}$. Not all of these coefficients can be in $\Sigma_{m}$. If they were, $J$ would be in $\Sigma_{m+1}$. Let $H$ be a coefficient which is not in $\Sigma_{m}$.

We say that any solution $\bar{u}_{i} ; \bar{y}_{1}, \cdots, \bar{y}_{m}$ of $\Sigma_{m}$ which does not annul $H$ can be completed into a solution of $\Sigma_{m+1}$ which does not annul $L$.

Let $a$ be a value of $x$ at which all functions of $x$ which we shall use are analytic and at which the above solution of $\Sigma_{m}$ does not annul $H$. Let the solution be substituted into $J$ and let numerical values then be attributed to the $y_{m+1, j}$ with $j<r_{m+1}$ in such a way as to give $J$ a numerical value, for $x=a$, which is not zero. We can then find a numerical value for the $r_{m+1}$ th derivative of $y_{m+1}$ which, together with $x=a$, etc., annuls $A_{m+1}$. Referring to $M$ in (5), we see that, because the remainder of $M$ with respect to (4) is zero and $I_{1}, \cdots, I_{m+1}$ do not vanish for the indicated numerical values, the values cause $M$ to vanish. Hence $L S_{m+1}$ does not vanish for the values. This means

(4) The $u_{i j}$ in II are those appearing in (4) and in $L$. 
that the above solution of $\Sigma_{m}$ can be completed into a regular solution of (4) which does not annul $L$, so that our statement is proved. We note that the $y_{i j}$ in $H$ have $j \leqq r_{i}, i=1, \cdots, m$.

8. We might have taken $L=1$ in $\S 7$. On this basis, let $K$, a form not in $\Sigma_{m+1}$ which involves only such $y_{i j}$ as appear in (4), be such that every solution of $\Sigma_{m+1}$ which does not annul $K$ can be completed into a solution of $\Sigma_{m+2}$. If, returning to $\Sigma_{m}$, we take $L=K$, we find an $H$ such that the solutions of $\Sigma_{m}$ which do not annul $H$ can be completed into solutions of $\Sigma_{m+2}$. The proof of the theorem stated in $\$ 4$ is thus easy to conclude.

\section{SUMS, PRODUCTS AND DERIVATIVES}

9. Let $\Sigma_{1}$ and $\Sigma_{2}$ be systems of forms in $y$, each system possessing solutions. It is possible to form, in various ways, sums $y^{\prime}+y^{\prime \prime}$ where $y^{\prime}$ and $y^{\prime \prime}$, solutions respectively of $\Sigma_{1}$ and of $\Sigma_{2}$, have the same area of analyticity. The manifold of the system of those forms in $y$ which vanish for all sums $y^{\prime}+y^{\prime \prime}$ will be called the arithmetic sum of the manifolds of $\Sigma_{1}$ and $\Sigma_{2}$. We define similarly arithmetic product, using all products $y^{\prime} y^{\prime \prime}$.

Let $\Sigma$ be a system of forms in $y$ which possesses solutions. There are forms in $y$ which vanish if $y$ is the derivative of any solution of $\Sigma$. The manifold of the totality of such forms will be called the derivative of the manifold of $\Sigma$.

Examples. If $\Sigma_{1}$ and $\Sigma_{2}$ are the forms $y_{1}-1$ and $x y_{1}-y$ respectively, the arithmetic sum of their manifolds is the two-parameter family of functions $y=a x+b$. The arithmetic product is the family $a x^{2}+b x$. The derivative of the manifold of $\Sigma_{2}$ is the manifold of $y_{1}$.

10. Certain solutions in the sum of two manifolds may not be sums $y^{\prime}+y^{\prime \prime}$. Such special solutions will now be examined.

Let $\Sigma_{1}$ be a nontrivial closed system of forms in the unknown $u$. Let $\Sigma_{2}$ be a similar system in $v$. Let $\Lambda$ be a system in $u, v, y$ consisting of the forms in $\Sigma_{1}$, those in $\Sigma_{2}$ and $y-(u+v)$. Let $\Omega$ be the totality of forms in $u, v, y$ which hold $\Lambda$. One can prove that $\Omega$ contains nonzero forms in $y$ alone. Let $\Sigma^{\prime}$ be the totality of forms in $\Omega$ which are free of $u$ and $v$. If $\Omega$ is the intersection of closed irreducible systems $\Omega_{1}, \cdots, \Omega_{s}$, then $\Sigma^{\prime}$ will be the intersection of those subsystems of the $\Omega_{i}$ which are free of $u$ and $v$.

We refer now to $\$ 4$. We see that there is a nonzero form $G$ in $y$ alone, holding no essential irreducible manifold in the manifold of $\Sigma^{\prime}$, which is such that every solution of $\Sigma^{\prime}$ which does not annul $G$ can be represented, in some area, as the sum of a solution of $\Sigma_{1}$ and a solution of $\Sigma_{2}$.

Let us apply these conclusions to the systems $\Sigma_{1}$ and $\Sigma_{2}$ of $\$ 9$, which we shall suppose closed and nontrivial, with the respective manifolds $\mathfrak{M}_{1}$ and $\mathfrak{M}_{2}$ of sum $\mathfrak{M}$. Let $G$ be a form in $y$, holding no essential irreducible manifold in $\mathfrak{M}$, which is such that every solution in $\mathfrak{M}$ which does not annul $G$ is the sum of solutions taken from $\mathfrak{M}_{1}$ and $\mathfrak{M}_{2}$. 
Let $\bar{y}$ be any solution in $\mathfrak{M}$. Let $\mathfrak{A}^{\prime}$ be any area in which $\bar{y}$ is analytic. Let $m$ be a positive integer and $\epsilon$ a positive number. Let $\tilde{y}$ be a solution in $\mathfrak{M}$, analytic in an area $\mathfrak{A}_{1}$ contained in $\mathfrak{A}^{\prime}, \tilde{y}$ being so taken that $G$ is not annulled by $\tilde{y}$ at any point of $\mathfrak{A}_{1}$ and that $\dot{y}-\bar{y}$ has at each point of $\mathfrak{A}_{1}$ a Taylor expansion in which the first $m+1$ coefficients are of moduli less than $\epsilon$. The existence of $\tilde{y}$ is obvious. Let $\mathfrak{A}_{1}^{\prime}$ be an area contained in $\mathfrak{A}_{1}$ in which $\tilde{y}$ is the sum of solutions taken from $\mathfrak{M}_{1}$ and $\mathfrak{M}_{2}$. We now find a second $\tilde{y}$, using an area $\mathfrak{A}_{2}$ in $\mathfrak{A}_{1}{ }^{\prime}$, a larger $m$ and a smaller $\epsilon$. Continuing, we see that there exists a set of points, dense in the area in which $\bar{y}$ is analytic, such that, given any point a of the set, any positive integer $m$ and any $\epsilon>0$, there is a solution $\tilde{y}$ in $\mathfrak{M}$ which, for the neighborhood of $a$, is the sum of solutions taken from $\mathfrak{M}_{1}$ and $\mathfrak{M}_{2}$, the first $m+1$ coefficients in the expansion of $\bar{y}-\bar{y}$ at a being of moduli less than $\epsilon$.

A similar result holds for the product of $\mathfrak{M}_{1}$ and $\mathfrak{M}_{2}$.

\section{Description of Results of Painlevé}

11. In $\$ 12$ we shall employ results of Painlevé concerning the algebroid character of the solution of an algebraic differential equation of the first $\operatorname{order}\left({ }^{5}\right)$. While these results have received enough attention to warrant describing them as classic, they have not thus far, to our knowledge, been given didactic exposition. Here, we shall limit ourselves to formulating Painlevé's results in a manner which will permit us to employ them with precision $\left(^{6}\right)$.

Let $A$ be an algebraically irreducible form in $y$ of the first order, of degree $n$ in $y_{1}$. Let $\mathfrak{A}$ be the area in which the coefficients in $A$ are meromorphic. There figures, in the statement of Painlevé's results, a set of points $\varepsilon$, contained in $\mathfrak{A}$, which includes the poles of the coefficients in $A$ and has no limit point in the interior of $\mathfrak{A}$. When $\mathcal{E}$ is removed from $\mathfrak{A}$, there remains an open region $\mathfrak{A}^{\prime}$.

Let $x_{0}$ be any point of $\mathfrak{U}^{\prime}$. Let $b$ be any finite number. Then, given any number $y_{0}$, close to $b$ and distinct from $b, A$ has precisely $n$ distinct solutions analytic at $x_{0}$ and assuming the value $y_{0}$ at $x_{0}$.

There exist, furthermore, a certain number $j$ (depending on $x_{0}$ and $b$ ) of equations

$$
y^{m_{i}}+\alpha_{1 i}\left(x, y_{0}\right) y^{m_{i}-1}+\cdots+\alpha_{m_{i} i}\left(x, y_{0}\right)=0, \quad i=1, \cdots, j,
$$

whose descriptions and roles are as follows. The $\alpha_{k i}$ are functions of $x$ and $y_{0}$, analytic for $\left|x-x_{0}\right|<\delta,\left|y_{0}-b\right|<\delta$, where $\delta$ is some positive number depending on $x_{0}$ and $b$. For $y_{0}$ close to $b$ and distinct from $b$, each of the $n$ solu-

(5) Painlevé, Lȩ̧ons sur la Theorie des Équations Différentielles Professées a Stockholm, Paris, 1897, pp. 70-76.

(8) The matter is not very difficult to work out, starting with the indications given by Painelvé. It is helpful to read Schlesinger, Gewöhnliche Differenzialgleichungen, Chapter 3, where somewhat related questions are considered. The Weierstrass preparation theorem can be employed to advantage. 
tions of $A$ mentioned above satisfies one of the equations (6) in the neighborhood of $x=x_{0}$. Furthermore, every solution in the general solution( $\left(^{7}\right)$ of $A$ which is analytic at $x_{0}$ and assumes the value $b$ at $x_{0}$ satisfies one of the equations (6). Again, if $y(x)$ is a function analytic in an area contained in $\left|x-x_{0}\right|<\delta$ and if $y(x)$ satisfies one of the equations (6) with $y_{0}$ fixed at a value interior to a circle of center $b$ and radius $\delta$, then $y(x)$ is a solution in the general solution of $A$. For a given $y_{0}$ close to $b,(6)$ may yield, in addition to solutions of $A$ which equal $y_{0}$ at $x_{0}$, other solutions of $A$ analytic at $x_{0}$.

We now deal with solutions of $A$ which assume large values at $x_{0}$. There exists a $g>0$ such that, for $\left|y_{0}\right|>g, A$ has precisely $n$ distinct solutions, analytic at $x_{0}$, which assume the value $y_{0}$ at $x_{0}$. There exists a number $h$ (independent of $x_{0}$ ) of equations

$$
z^{p_{i}}+\beta_{1 i}\left(x, z_{0}\right) z^{p_{i}-1}+\cdots+\beta_{p_{i} i}\left(x, z_{0}\right)=0, \quad i=1, \cdots, h,
$$

with $\beta_{k i}$ which are analytic for $x=x_{0}, z_{0}=0$. Given any solution $y$ of $A$, analytic at $x_{0}$ and assuming there a large value $y_{0}$, the function $z=1 / y$ satisfies one of the equations (7) with $z_{0}=1 / y_{0}$. Given any function $z$ distinct from zero, obtained from the equations (7) for a small value of $z_{0}$, the reciprocal of $z$ is a solution of $A$.

We proceed to apply these results of Painlevé.

\section{LIMITED SUMS AND PRODUCTS}

12. We prove the following theorem.

Theorem I. Let $\mathfrak{M}_{1}$ and $\mathfrak{M}_{2}$ be general solutions of forms of the first order in $y$. Let $\mathfrak{M}_{1}$ and $\mathfrak{M}_{2}$ be limited. Then the sum and the product of $\mathfrak{M}_{1}$ and $\mathfrak{M}_{2}$ are limited manifolds.

We take first the case of the product, disposing of that case by establishing the following result $\left({ }^{8}\right)$.

THEOREM II. Let $\mathfrak{M}_{1}$ and $\mathfrak{M}_{2}$ be general solutions of forms of the first order. Let neither $\mathfrak{M}_{1}$ nor $\mathfrak{M}_{2}$ have zero among its solutions. Then zero is not a solution in the product of $\mathfrak{M}_{1}$ and $\mathfrak{M}_{2}$.

Let us assume that zero is in the product. There are values of $x$ at which zero can be approximated, as in $\$ 10$, by products of solutions in $\mathfrak{M}_{1}$ and $\mathfrak{M}_{2}$. We select a value $x_{0}$ of this type which does not belong to either of the sets $\varepsilon$

$\left.{ }^{7}\right)$ The notion of general solution, as employed here, does not, of course, appear in Painlevé's work.

${ }^{8}$ ) What is involved here is the following. Let $\mathfrak{M}$ be the product of the limited $\mathfrak{M}_{1}$ and $\mathfrak{M}_{2}$ of Theorem I. By $\S 1$, the reciprocals of the solutions distinct from zero in $\mathfrak{M}_{1}$ and $\mathfrak{M}_{2}$ are manifolds. We represent the manifolds of reciprocals, which are seen without trouble to be general solutions of forms of the first order, by $\mathfrak{M}_{1}^{\prime}$ and $\mathfrak{M}_{2}^{\prime}$ and their product by $\mathfrak{M}^{\prime}$. A form $F$ holds $\mathfrak{M}^{\prime}$ if it vanishes for every $1 /\left(y^{\prime} y^{\prime \prime}\right)$ with $y^{\prime}$ in $\mathfrak{M}_{1}$ and $y^{\prime \prime}$ in $\mathfrak{M}_{2}$. By $\$ 10, F$ will vanish for the reciprocal of every nonzero solution in $\mathfrak{M}$. Thus, if $\mathfrak{M}$ is not limited, $\mathfrak{M}^{\prime}$ contains zero. 
of $\$ 11$ associated with the forms whose general solutions are $\mathfrak{M}_{1}$ and $\mathfrak{M}_{2}$.

For convenience, we use $y$ to designate solutions in $\mathfrak{M}_{1}$ and $u$, similarly, for $\mathfrak{M}_{2}$. Let there be given a sequence of $y u$ whose expansions tend toward zero at $x_{0}$. From this sequence we can select a subsequence in which the values $y\left(x_{0}\right), u\left(x_{0}\right)$ tend toward definite limits, finite or infinite. We may thus, and shall, assume that such limits exist for the given sequence. We assume, as we may, that the limit of the $y\left(x_{0}\right)$ is zero. The limit of the $u\left(x_{0}\right)$ will be a quantity $c$, finite or infinite.

We treat first the case in which $c$ is finite.

We may suppose that all of the $y$ satisfy a single equation (6). We write this equation here in the form

$$
y^{m}+\alpha_{1}\left(x, y_{0}\right) y^{m-1}+\cdots+\alpha_{m}\left(x, y_{0}\right)=0 .
$$

Similarly, the $u$ may be supposed to satisfy an equation

$$
u^{n}+\beta_{1}\left(x, u_{0}\right) u^{n-1}+\cdots+\beta_{n}\left(x, u_{0}\right)=0 .
$$

Because zero is not a solution in $\mathfrak{M}_{1}, \alpha_{m}$ cannot vanish identically in $x$ for a small value of $y_{0}$; similarly, $\beta_{n}$ cannot vanish in $x$ for a value of $u_{0}$ close to $c$.

The theory of symmetric functions shows that the $y u$ satisfy an equation

$$
(y u)^{m n}+\gamma_{1}(y u)^{m n-1}+\cdots+\gamma_{m n}=0
$$

where the $\gamma$ are polynomials in the $\alpha$ and the $\beta$, with $\gamma_{m n}=\alpha_{m}^{n} \beta_{n}^{m}$. Because the Taylor expansions of the $y u$ approach zero, (10) must be satisfied, for $y_{0}=0$, $u_{0}=c$, by $y u=0$. This is not so. We have thus disposed of the case finite.

Now, suppose that $c=\infty$. We let $z$ represent the reciprocals of the $u$. We may assume that the $z$ all satisfy an equation

$$
z^{p}+\cdots+\delta_{p}\left(x, z_{0}\right)=0 .
$$

Then the $y / z$ satisfy an equation

$$
\phi_{0}(y / z)^{m p}+\cdots+\phi_{m p}=0
$$

with $\phi$ which are polynomials in the $\alpha$ and $\delta$, and with, in particular,

$$
\phi_{0}=\delta_{p}^{m}, \quad \phi_{m p}=\alpha_{m}^{p} .
$$

We reach the contradiction that (12) is satisfied by $y / z=0$ for $y_{0}=z_{0}=0$. This concludes the proof of our statement in regard to products.

Continuing with Theorem I, we consider the limited $\mathfrak{M}_{1}$ and $\mathfrak{M}_{2}$, under the assumption that their sum is not limited.

Using $y$ for $\mathfrak{M}_{1}$ and $u$ for $\mathfrak{M}_{2}$, we consider an $x_{0}$, and a sequence of $y+u$ for which the expansions of the $1 /(y+u)$ tend toward the expansion of zero at $x_{0}\left({ }^{9}\right)$. We shall assume, furthermore, that the sequences of values $y\left(x_{0}\right)$,

(9) That such a sequence exists can be shown without difficulty by the method of $\S 10$. 
$u\left(x_{0}\right)$ tend toward definite limits, finite or infinite. At least one of these limits is infinite. Let this be so for the $u\left(x_{0}\right)$. We suppose first that the $y\left(x_{0}\right)$ have a finite limit.

We arrange so that the $y$ satisfy an equation (8) and the reciprocals $z$ of the $u$ an equation (11). Let

$$
w=\frac{1}{y+u}=\frac{z}{1+y z} .
$$

We find the $w$ to satisfy an equation $\phi_{0} w^{m p}+\cdots+\phi_{m p}=0$ with $\phi_{m p}=\delta_{p}^{m}$. We must thus have $\delta_{p}(x, 0)=0$. This produces the contradiction that $\mathfrak{M}_{2}$ is not limited. The case in which the $y\left(x_{0}\right)$ approach $\infty$ is handled in much the same way.

\section{EQUATIONS OF HIGHER ORDER}

13. We shall show by means of examples suggested by the theory of the elliptic functions that the above results cannot be extended to equations of the second order.

The nonconstant solutions of

$$
y_{1}^{2}=4\left(y^{3}-e^{3}\right),
$$

where $e$ is any constant, satisfy the equation

$$
y_{2}-6 y^{2}=0,
$$

whose manifold $\mathfrak{M}_{1}$ is, by $\S 3$, limited. If, in (13), we replace $y$ by $y+e,(13)$ goes over into

$$
y_{1}^{2}=4\left(y^{3}+3 e y^{2}+3 e^{2} y\right)
$$

which implies, when $y$ is not a constant,

$$
16 y^{6}-8 y^{3} y_{1}^{2}-8 y^{4} y_{2}+y_{1}^{4}-4 y y_{1}^{2} y_{2}+4 y^{2} y_{2}^{2}=0
$$

with a limited manifold $\mathfrak{M}_{2}$. For any constant $e$, arbitrarily large, there are solutions in $\mathfrak{M}_{1}$ and $\mathfrak{M}_{2}$ respectively whose difference is $e$. This is enough to show that the theorem on sums does not hold for equations of the second order.

If in (14), we replace $y$ by $3 e^{2} / y$, (14) remains invariant. Thus, for any $e$, (15) has two solutions whose product is $3 e^{2}$. The theorem on the product thus does not carry over to the second order.

\section{The DeRIVATIVE}

14. We prove the following theorem.

THEOREM. The derivative of a limited manifold is limited. 
Let $\mathfrak{M}$, limited, be held by a form $F=y^{p}-G$ with every term in $G$ of degree less than $p$. We have $y^{p} \equiv G,(F)$. Now $y_{1}^{2 p-1} \equiv 0,\left(y^{p}\right)$. Hence there is a relation $y_{1}^{2 p-1} \equiv H,(F)$ with every term in $H$ of degree less than $2 p-1$.

We arrange $y^{p}-G$ and $y_{1}^{2 p-1}-H$ in powers of $y$, securing two polynomials in $y$,

$$
A_{0} y^{p}+\cdots+A_{p}
$$

and

$$
B_{0} y^{q}+\cdots+B_{q}
$$

Here $A_{0}=1$ and $A_{i}$ is of degree less than $i$ for $i>0$. Also, $B_{q}$ has $y_{1}^{2 p-1}$ as one term and its other terms are of degree less than $2 p-1$. Each $B_{i}$ with $i<q$ is of degree less than $2 p-q+i-1$.

We consider the resultant, $R$, of (16) and (17) with respect to $y$. One of the terms of $R$ is $A_{0}^{q} B_{q}^{p}$, that is, $B_{q}^{p}$. Now $B_{q}^{p}$ contains $y_{1}^{(2 p-1) p}$ and its other terms are of degree less than $(2 p-1) p$. Consider any other term in $R$,

$$
T=k A_{\mu_{1}} \cdots A_{\mu_{q}} B_{\nu_{1}} \cdots B_{\nu_{p}} .
$$

We have

$$
\mu_{1}+\cdots+\mu_{q}+\nu_{1}+\cdots+\nu_{p}=p q .
$$

At least one $\mu$ is positive and at least one $\nu$ is less than $p$. We have thus, for the degree $d$ of $T$ in the $y_{i}$,

$$
d<\sum \mu_{i}+\sum\left(2 p-q+\nu_{i}-1\right)=(2 p-1) p .
$$

Thus $R=y_{1}^{(2 p-1) p}+K$ where each term in $K$ is of degree less than $(2 p-1) p$. We note that the $y_{i}$ in $K$ have $i>0$. Now $R$ holds $\mathfrak{M}$. Then the derivative of $\mathfrak{M}$ is held by the form obtained from $R$ by replacing each $y_{i}$ appearing in $R$ by $y_{i-1}$. Thus the derivative of $\mathfrak{M}$ is limited.

15. We shall prove that, if $F$, in $\$ 14$, is of the first order, the derivative of $\mathfrak{M}$ is held by a form $y^{q}+L$ with $L$ of the first order and of degree less than $q$.

It will suffice to prove that the derivative of the manifold of $F$ is held by a form $y^{q}+L$ as just described. In that proof, we may and shall assume that $F$ is algebraically irreducible.

We consider $F$ and its derivative $F_{1}$ as polynomials in $y$ and denote their resultant with respect to $y$, which is not identically zero, by $R$. Now $R$ must involve $y_{2}$ effectively; otherwise $R$, which holds $F$, would be divisible by $F$, which involves $y$.

We show now that $R$ contains a term in $y_{1}$ alone which is of higher degree than any other term in $R$. This will prove our statement.

Let us assume that the terms of highest degree in $R$ involve $y_{2}$. We consider the equation $R \doteq 0$ as an algebraic equation for $y_{2}$. It will be satisfied, 
for the neighborhood of $y_{1}=\infty$, by some series of descending rational powers of $y_{1}$,

$$
y_{2}=\alpha y_{1}^{s / r}+\beta y_{1}^{(8-1) / r}+\cdots
$$

with $r>0, s \leqq r$ and $\alpha, \beta$, etc., functions of $x$ analytic in some area $\left({ }^{10}\right)$.

We substitute this expression for $y_{2}$ into $F_{1}$, whereupon $F_{1}$ goes over into a polynomial $f$ in $y$ whose coefficients are infinite series in $y_{1}$. We consider the equations $F=0$ and $f=0$ as algebraic equations for $y$. They must have a common solution given by a series of descending powers of $y_{1}$, effectively involving $y_{1}$,

$$
y=\phi y_{1}^{t / u}+\cdots
$$

with $u$ a multiple of $r,(19)$ converging for large values of $y_{1}$. We assume that $\phi \neq 0$.

Suppose first that $t>0$. Then $t<u$ since, when $F$ is considered as a polynomial in $y$ and $y_{1}$, its term $y^{p}$ is of higher degree than every other term. From (19), we find by inversion, for the neighborhood of $y=\infty$, a series of descending powers for $y_{1}$ of the type

$$
y_{1}=\psi y^{u / t}+\cdots .
$$

Substituting $y_{1}$, as in (20), into (18), we find a series for $y_{2}$

$$
y_{2}=\lambda y^{s u / r t}+\cdots \text {. }
$$

If we replace $y_{1}$ and $y_{2}$ in $F_{1}$ by their expressions in (20) and (21), $F_{1}$ will vanish identically in $x$ and $y$. But if we replace $y_{1}$ in the equation $F_{1}=0$ by the second member of (20) and solve the resulting equation for $y_{2}$, we will find for $y_{2}$ a series in $y$ obtained by differentiating the second member of (20) and replacing $y_{1}$ in the result by its expression in (20). The series thus obtained begins effectively with a power of $y$ whose exponent is $(2 u / t)-1$, which exceeds the first exponent in (21).

Now suppose that $t=0$. Then (19) yields an expansion for $y_{1}$ in ascending powers of $y-\phi$ of the type

$$
y_{1}=\mu(y-\phi)^{-k}+\cdots
$$

where $k$ is a positive rational number. Substituting (22) into (18) and proceeding as above, we find again a contradiction of the fact that $s<r$.

The case of $t<0$ is handled in the same way.

16. If $F$ is of order $r>1$, we cannot infer that the derivative of $\mathfrak{M}$ is held by a form $y^{q}+L$ with $L$ of order not higher than $r$ and of degree less than $q$. Let $\mathfrak{M}$ be the manifold of $y_{2}-y^{2}$. We find that $\mathfrak{M}$ is held by

(10) The second member of (18) may be zero. 


$$
A=y_{3}^{2}-4 y_{2} y_{1}^{2} \text {. }
$$

Suppose now that $\mathfrak{M}$ is held by $B=y_{1}^{q}+K$ where $K$, free of $y$, is of order not higher than 3 and has no term of degree as high as $q$. Because $y_{1}^{q}$ is effectively a term in $B$ and is divisible neither by $y_{2}$ nor $y_{8}, B$ is not divisible by $A$. Hence, the resultant $R$ of $A$ and $B$ with respect to $y_{3}$ is a nonzero polynomial $R$ in $y_{1}$ and $y_{2}$. Putting $y_{2}=y^{2}$ in $R$, we find the contradiction that $\mathfrak{M}$ is held by a form of order less than 2 .

Columbia University, NEw YORK, N: Y. 